\title{
Effect of powder size and processing parameters on surface, density and mechanical properties of 316L elaborated by Laser Powder Bed Fusion
}

\author{
Sabrine Ziri, Anis Hor and Catherine Mabru
}

\author{
Sabrine Ziri. Institut Clément Ader (ICA) ; Université de Toulouse ; CNRS, IMT Mines Albi, INSA, ISAE-SUPAERO, UPS, 3 rue \\ Caroline Aigle, 31400 Toulouse, France. \\ Corresponding author: Ziri Sabrine. E-mail address: Sabrine.ziri@isae-supaero.fr \\ Anis Hor. Institut Clément Ader (ICA) ; Université de Toulouse ; CNRS, IMT Mines Albi, INSA, ISAE-SUPAERO, UPS, 3 rue Caroline \\ Aigle, 31400 Toulouse, France. \\ Catherine Mabru. Institut Clément Ader (ICA) ; Université de Toulouse ; CNRS, IMT Mines Albi, INSA, ISAE-SUPAERO, UPS, 3 rue \\ Caroline Aigle, 31400 Toulouse, France.
}

Abstract. Despite the attractive capabilities of additive manufacturing (AM) technology, the industrialization of these processes remains very low. This is attributed to the complexes physical phenomena involved in the AM process and the layered structure of the produced parts. Intense research work is still needed for the prediction and optimization of AM parts mechanical properties. In this study, the influence of particle size distribution (PSD) of stainless steel 316L (SS 316L) powders on AM parts properties was investigated. Four PSD were used to produce test parts and compare the resulting porosity, surface roughness and macro-hardness. The SS 316L specimens were fabricated by Laser Powder Bed Fusion process (LPBF) on a SLM 125HL machine using variations in laser power and scan velocity. Computed scan tomography (CT) was used to characterize the defects. Lack of fusion and keyhole defects were detected. Defects were detected even in nearly dense parts. The powder size distribution was found to affect the porosity. Results from CT tests were used to identify the minimum achievable porosities for each powder, through the appropriate selection of process parameters. The macro-hardness and surface roughness were found to vary with the powder properties.

Keywords. Laser Powder Bed Fusion, Powder Size, Porosity, Surface Roughness, Hardness

\section{Introduction}

Additive manufacturing (AM) is a promising technology enabling the fabrication of parts with customizable designs, near-net shape geometries and high mechanical properties using various materials including metallic, ceramic and polymer materials $[1,2]$. Yet, there are significant challenges slowing down the progress of the AM technology. Among, these challenges the build-to-build variations of AM parts properties [3].

In Laser Powder Bed Fusion (LPBF) process, dense parts can be produced with high dimensional accuracy. The quality of the final part is influenced by the powder characteristics and the chosen processing parameters [4-6]. Several studies have confirmed that the particle size distribution (PSD), flowability and morphology of metallic powder material have a major impact on the density of final parts. It is possible to obtain high density values with different powder sizes, yet, the process parameters have to be adjusted accordingly. Gürtler et al. [7] studied the effect of AlSi12Mg PSD on melt pool dynamics and porosity formation to detect the defects on AM part. Their study showed that powder mixtures with more and smaller particles can compensate the defects in powder bed and improve parts density. They demonstrated the importance of defectless and homogenous layer coating for the production of defectless parts. Liu et al. [8] investigated the effect of laser and SS 316L powder properties on final AM part performance. They obtained higher density parts with smoother side surfaces using powder with wider range of particle size, whereas, the powder with narrower range of particle size provided higher ultimate tensile strength and larger hardness. Balbaa et al. [9] studied the influence of AlSi10Mg powder particle size on the LPBF processibility by considering the material physical properties. They used fine $(\mathrm{D} 50=9 \mu \mathrm{m})$ and coarse $(\mathrm{D} 50=40 \mu \mathrm{m})$ powders. The coarse powders, with higher flowability 
Effect of powder size and processing parameters on surface, density and mechanical prop...

and packing density, led to lower top surface roughness and higher microhardness. Therefore, the use of different powder types alters the surface finish and the mechanical properties of AM parts.

The aim of this work is to establish a relationship between powder properties and process parameters for Stainless Steel (SS) 316L to obtain full dense parts with good surface finish and high mechanical properties. Four powder sizes were used which are fine powder $\mathrm{D}_{50}=10 \mu \mathrm{m}$, medium powders $\mathrm{D}_{50}=29 \mu \mathrm{m}$ and $\mathrm{D}_{50}=41 \mu \mathrm{m}$ and coarse powder $\mathrm{D}_{50}=73 \mu \mathrm{m}$. The powders differ in flowability, relative density, chemical composition and mean powder diameter. The influence of powder properties on the porosity, surface roughness and macro-hardness was discussed. The observed trends were explored based on process parameters and powder size, flowability and composition.

\section{Material and methods}

\subsection{L-PBF Machine and processing parameters}

The AM samples were produced using the SLM 125HL machine, equipped with a single fiber laser of $400 \mathrm{~W}$. In order to fabricate AM dense parts with lowest surface roughness and highest mechanical properties, a parametric study is done to optimize the most influencing parameters. The samples were fabricated using a variation of processing parameters including laser power P and scan velocity V. The hatch distance $\mathrm{h}$ and layer thickness $\mathrm{L}$ were kept constants $(\mathrm{h}=120 \mu \mathrm{m}$ and $\mathrm{L}=50 \mu \mathrm{m}$ ). The build platform was preheated up to $100^{\circ} \mathrm{C}$. The scan strategy consisted of stripes with $33^{\circ}$ rotation between successive layers.

The processing parameters were chosen to span around the recommended SLM standard values for SS 316L, as shown Table 1. The recommended SLM values for SS $316 \mathrm{~L}$ are $\mathrm{P}=275 \mathrm{~W}, \mathrm{~V}=700 \mathrm{~mm} / \mathrm{s}, \mathrm{L}=50 \mu \mathrm{m}$ and $\mathrm{h}=120 \mu \mathrm{m}$. In total, nine sets of parameters were used to produce the samples for each type of SS 316L powder material. The parameters were chosen for the purpose of understanding the effect of processing parameters and powder size on AM parts properties. The energy density $E=P / V L h$ is a common variable used to determine the operating window for dense parts. It includes all the above mentioned parameters [10]: the laser power $(\mathrm{P})$, the scan velocity $(\mathrm{V})$, the hatch distance $(\mathrm{h})$ and the layer thickness (L).

Table 1. The processing parameters used to produce the samples

\begin{tabular}{c|c|c|c|c}
\hline Power, P(w) & Velocity. V(mm/s) & hatch distance, h(mm) & layer thickness, L(mm) & Energy density, E(J/mm $\left.{ }^{3}\right)$ \\
\hline 200 & 1000 & 0.12 & 0.05 & 33,3 \\
\hline 275 & 1000 & 0.12 & 0.05 & 45,8 \\
\hline 200 & 700 & 0.12 & 0.05 & 47,6 \\
\hline 350 & 1000 & 0.12 & 0.05 & 58,3 \\
\hline $\mathbf{2 7 5}$ & $\mathbf{7 0 0}$ & $\mathbf{0 . 1 2}$ & $\mathbf{0 . 0 5}$ & $\mathbf{6 5 , 5}$ \\
\hline 200 & 400 & 0.12 & 0.05 & 83,3 \\
\hline 350 & 700 & 0.12 & 0.05 & 83,3 \\
\hline 275 & 400 & 0.12 & 0.05 & 114,6 \\
\hline 350 & 400 & 0.12 & 0.05 & 145,8 \\
\hline
\end{tabular}

\subsection{The SS 316L powder materials}

The samples were produced in SS 316L using four different particle size distributions, as given in Table 2. The particle size distribution was measured by laser diffraction. Our target was to test fine, medium and coarse powders. The powder of type3 was bought from SLM supplier. The remaining three powders were bought from Höganäs supplier. The same raw powder material was sieved to control PSD and obtain various powder types (fine, medium and coarse powders). The powders type 2 and type3 correspond to the SLM recommended PSD for SS 316L and 
they are characterized by Gaussian like grain size distribution with powder particles diameters below $50 \mu$ m. Type 4 is characterized by Gaussian like distribution and particles diameters higher than $50 \mu \mathrm{m}$. The fine powder which is type 1 consists of particles with diameters below $18 \mu \mathrm{m}$ and is characterized by an asymmetric distribution with an increased concentration of fine particles. In total, there are two categories of powder materials (SLM and Höganäs) with nominal composition provided in Table 3. The main differences between the powder categories compositions are the percentages of $\mathrm{Si}, \mathrm{Ni}$ and $\mathrm{Mn}$.

Table 2. The SS 316L powder materials used to produce the samples

\begin{tabular}{l|c|c|c|c}
\hline Type & Type 1 - H6 & Type2 - H27 & Type3 - S34 & Type4-H67 \\
\hline Manufacturer & Höganäs & Höganäs & SLM & Höganäs \\
\hline $\mathrm{D}_{10}$ & 4.95 & 19.3 & 30.1 & 53.3 \\
\hline $\mathrm{D}_{50}$ & 10.01 & 29.2 & 41.2 & 73 \\
\hline $\mathrm{D}_{90}$ & 16.3 & 43.1 & 56.2 & 100 \\
\hline Mean diameter $(\mu \mathrm{m})$ & 6 & 27.4 & 34.2 & 66.9 \\
\hline
\end{tabular}

Table 3. The chemical composition of two powder categories from SLM and Höganäs

\begin{tabular}{|c|c|c|c|c|c|c|c|c|c|c|c|c|}
\hline Elen & & Si & $\mathrm{S}$ & P & $\mathrm{O}$ & $\mathrm{N}$ & C & $\mathbf{N i}$ & Mo & Mn & $\mathrm{Cr}$ & $\mathrm{Fe}$ \\
\hline 4-Höganăs & t\%] & 0.2 & 0.004 & $<0.01$ & 0.04 & 0.01 & 0 & 11.9 & 2.3 & 1.5 & 17.7 & Balance \\
\hline Type3 - SLM & [wt\%] & 0.61 & 0.005 & 0.008 & 0.02 & 0.1 & 0.017 & 12.58 & 2.35 & 0.54 & 17.78 & Balance \\
\hline
\end{tabular}

The properties of the four powder types were determined and compared by evaluating their flowability and density. The apparent density was measured using Scott volumeter according to ASTM B329-06 standard. The measurements were repeated five times and were conducted at ambient temperature. The flowability was measured using Hall method according to ASTM B213 standard. The measurements were repeated three times and were conducted at ambient temperature. The results are reported in Table 4. All powder types have acceptable flowability except powder type1. The later powder has insufficient flowability with severe agglomerations. From the relative density, the thickness of the deposited powder can be calculated and it depends on powder size. During building, it increases till reaching a steady thickness which is known as the effective layer thickness Leff [4].

Table 4. Physical properties of different powder

\begin{tabular}{l|c|c|c|c}
\hline Type & Type 1-H6 & Type2 - H27 & Type3 - S34 & Type4-H67 \\
\hline Flowability Hall $(\mathrm{s})$ & No flow & 17.5 & 16.9 & 15 \\
\hline Apparent density $\left(\mathrm{g} / \mathrm{cm}^{3}\right)$ & 3.63 & 4.5 & 4.6 & 4.21 \\
\hline Relative density $(\%)$ & 45 & 56.3 & 57.7 & 52.6 \\
\hline Effective layer thickness, Leff $(\mathrm{mm})$ & 0.110 & 0.088 & 0.087 & 0.095 \\
\hline
\end{tabular}

\subsection{Characterization of produced samples: porosity, surface roughness and macro-hardness}

Two types of samples were produced which are cylinders with $10 \mathrm{~mm}$ length and $4 \mathrm{~mm}$ diameters for porosity measurements and cubes with $15 \mathrm{~mm}$ length for surface roughness and macro-hardness tests. The instruments used for the samples characterization are detailed below:

- The surface roughness of top and side surface of as-built cubic samples was evaluated using Alicona microscope which 
Effect of powder size and processing parameters on surface, density and mechanical prop...

generates $3 \mathrm{~d}$ surface topography, as shown in Fig. 1 . The surface roughness parameter calculated in this study is the arithmetic mean surface roughness $\left(\mathrm{S}_{\mathrm{a}}\right)$ according to ISO 25178-2 standard;
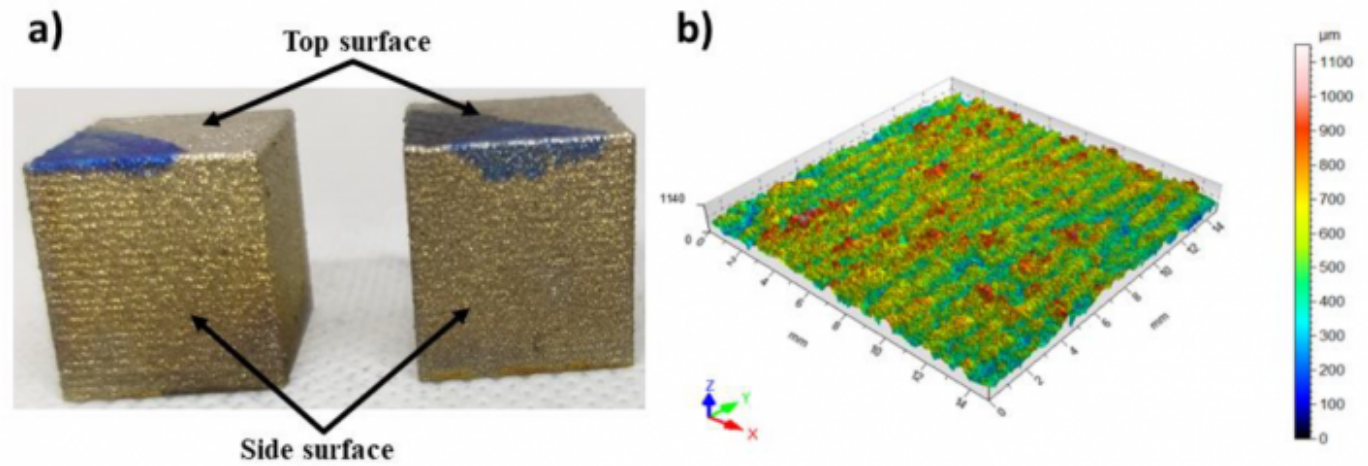

Fig. 1 Surface roughness testing: (a) Samples and (b) 3d surface topography exemple

- The porosity of cylindrical samples was measured using X-ray computed tomography method (CT). The samples were scanned to visualize the porosity distribution using a spatial resolution of $6.7 \mu \mathrm{m}$. The analyzed volume dimensions are $6 \mathrm{~mm}$ height and $3.9 \mathrm{~mm}$ diameter. The porosity results were confirmed by microscopic analysis and a maximum coefficient of variation of 5\% was obtained. For the microscopic analysis, an optical microscope was used and a total of 10 images located randomly were analyzed;

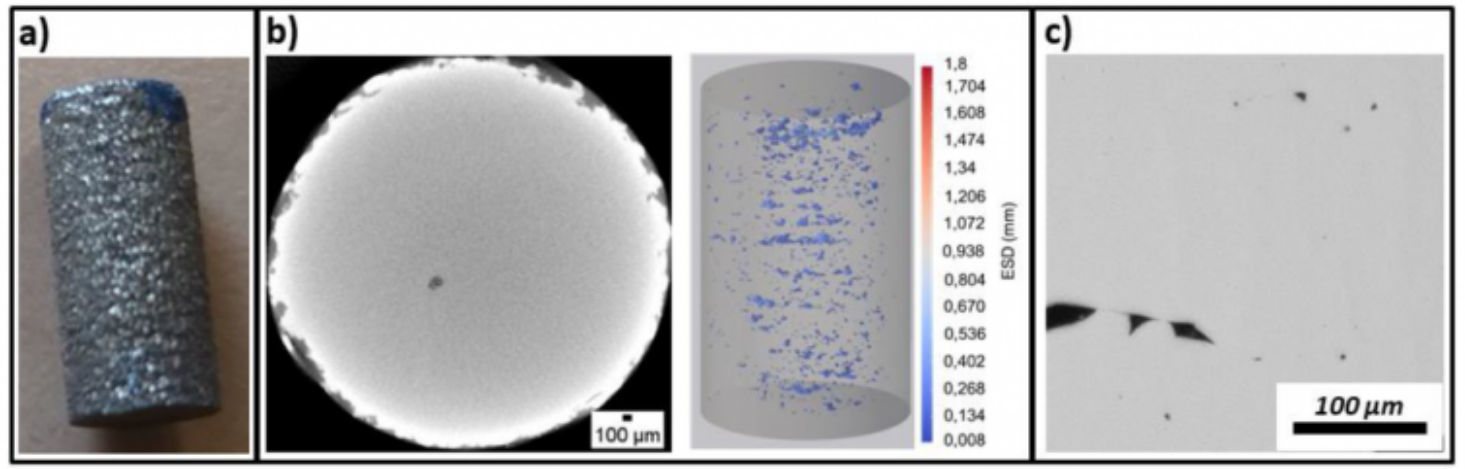

Fig. 2 Porosity measurement sample geometry and results: (a) Cylindrical sample, (b) Example of the obtained result by CT method and (c) Example of cross-section image by microscopic analysis

- As-polished samples were used for hardness testing. Macro-hardness measurements were conducted using a load of $30 \mathrm{Kg}$ being applied for 10s. The average of nine Vickers indents was determined for each sample. The indents were distant from the surface edges, visible porosities and previous test points. The hardness results were close for the same sample and no significant variation was observed either between side surface and top surface or along the length and width of the same surface. 


\section{Results and discussion}

\subsection{Surface roughness analysis}

The surface roughness of the AM samples depends on the chosen processing parameters and the layer coating system. It is important to evaluate the roughness of the top surface because it represents the quality of each scanned layer during building process. The roughness of the scanned layer affects the homogeneity of the spread powder layer which is influenced by powder properties (size [11], particle cohesiveness [11, 12]). Liu et al. [8] investigated the effect of particle size distribution on processing parameters optimization. They used two PSD: an OSPREY powder with wide range of particle size and mean size of $27.3 \mu \mathrm{m}$ and a LPW powder with narrow range and mean size of $29.3 \mu \mathrm{m}$. The Osprey powder generated smoother side surface finishing parts. They obtained significant variation in surface roughness particularly for the top surface. In this study, the comparison of surface roughness data from various powder types revealed that Sa of fine powder was higher compared to coarser counterparts, for top and side surface. The fine powder, characterized by poor flowability and high tendency of agglomeration, leads to rough and wavier surface as shown in Fig. 4. The medium powder types (type2 and type3) had lowest Sa as shown in Fig. 3. The surface for both types was generally free from un-melted or partially melted powder particles. During building process, the spatters ejection was more intense in coarse powder builds compared to fine or medium counterparts. The spattering phenomenon of coarse powder affects the surface quality of the samples and leads to rough surface $[11,13]$. There is a difference in surface roughness between side and top surface in the case of coarse powders.

a)

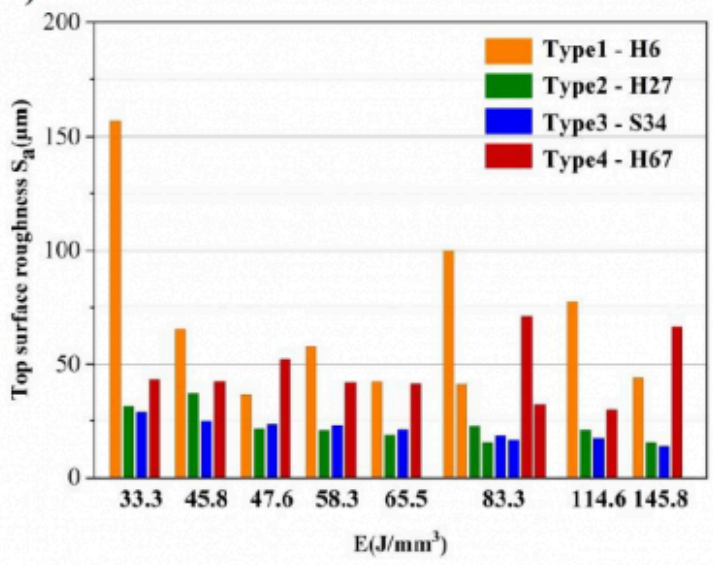

b)

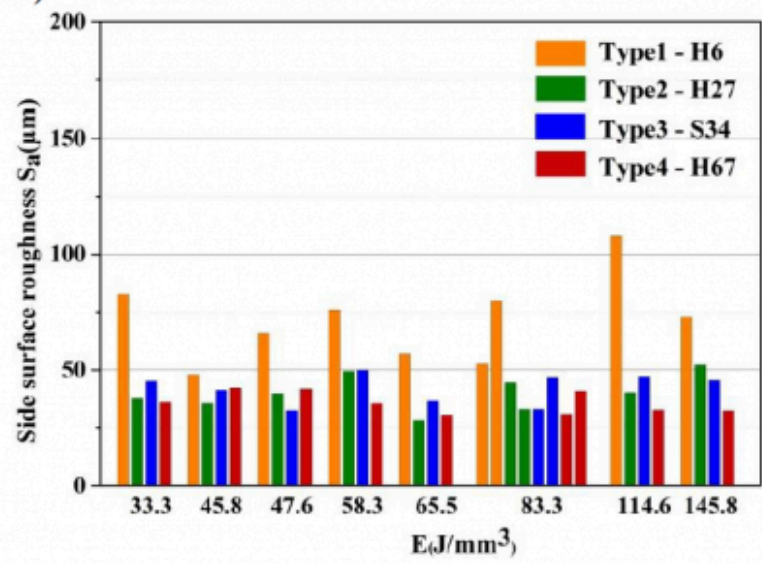

Fig. 3 Surface roughness as function of energy density for the four types of powder: (a) top surface and (b) side surface

\subsection{Porosity analysis}

For SS 316L samples fabricated with LPBF process, the density increases with the increasing energy density. It was reported that dense parts were produced using energy density close to $104.2 \mathrm{~J} / \mathrm{mm}^{3}$ [14-16]. Yakout et al. [14] found that stable melting occurs between $62.5-104.2 \mathrm{~J} / \mathrm{mm}^{3}$ energy densities for SS 316L. Above $104.2 \mathrm{~J} / \mathrm{mm}^{3}$, the density of the parts decreased due to vaporization of alloying elements. They used spherical SS 316L powder with diameter D50 $=29.1 \mu \mathrm{m}$. The PSD of the utilized powder is similar to powder type 2 in our study. The porosity results of our samples as function of the volumetric energy density is shown in Fig. 5. Highly dense samples with porosities below $0.1 \%$ were obtained for all powder types within various energy density ranges. The average porosity of samples 
Effect of powder size and processing parameters on surface, density and mechanical prop...

produced with fine powder type 1 was $5 \%$ higher than their counterparts fabricated with medium powders, whereas, the average porosity of samples fabricated with coarse powder type 4 was $1 \%$ higher than medium counterparts. In general, the porosity decreased when increasing the energy density and then stabilized for E higher than $83.3 \mathrm{~J} / \mathrm{mm}^{3}$. However within the applied energy density, no increase of the porosity at high energy densities was observed, contrary to what Yakout et al. [14] obtained. The dense medium powders samples (type2 and type3) were obtained for energy densities higher than $65.5 \mathrm{~J} / \mathrm{mm}^{3}$, whereas, the dense samples fabricated with coarse powder were obtained for energy densities above $114 \mathrm{~J} / \mathrm{mm}^{3}$. For fine powder, the dense sample was produced with E equal to $145 \mathrm{~J} / \mathrm{mm}^{3}$. For energies above $114 \mathrm{~J} / \mathrm{mm}^{3}$, the keyhole porosities or trapped gas defects are detected as shown in Fig. 6 . At low energy densities, irregular shaped defects and lack of fusion porosities were formed. This is mainly due to the uncomplete melting leading to the formation of defects containing un-melted or partially melted particles. 


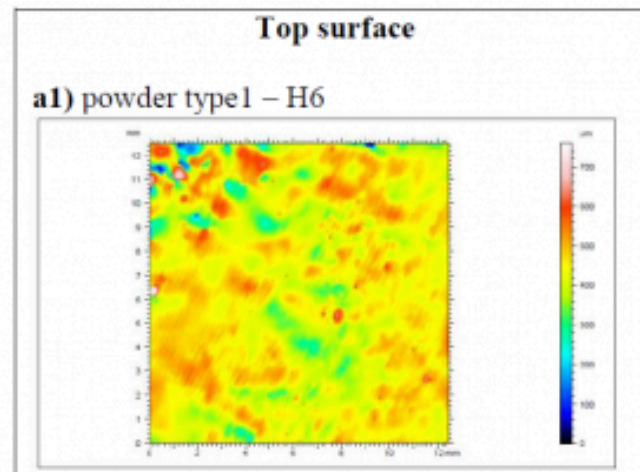

b1) powder type $2-\mathrm{H} 27$

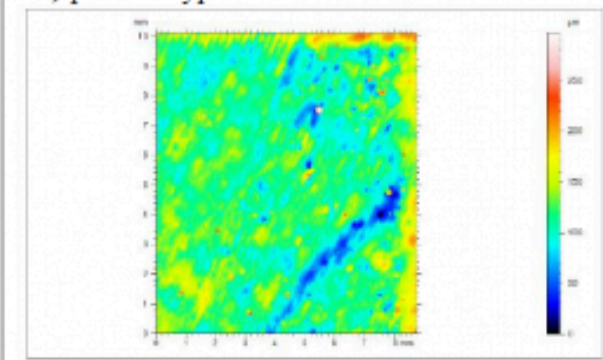

c1) powder type3-S34

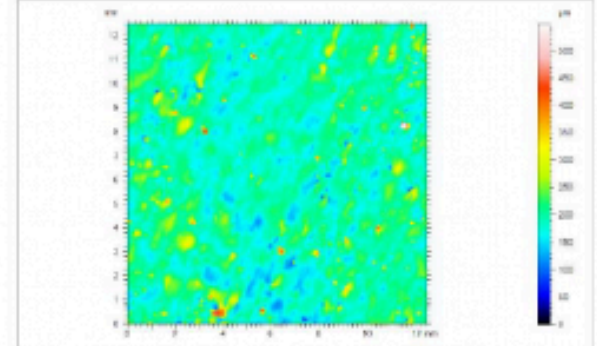

d1) powder type 4 - $\mathrm{H} 67$

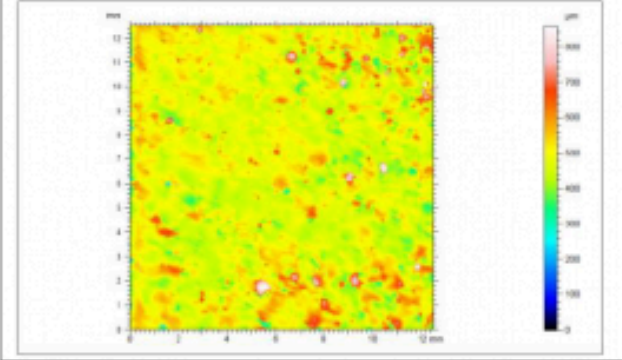

Side surface

a2) powder type $1-\mathrm{H} 6$

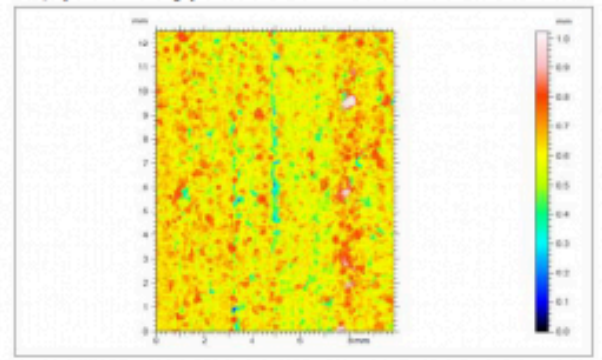

b2) powder type $2-\mathrm{H} 27$

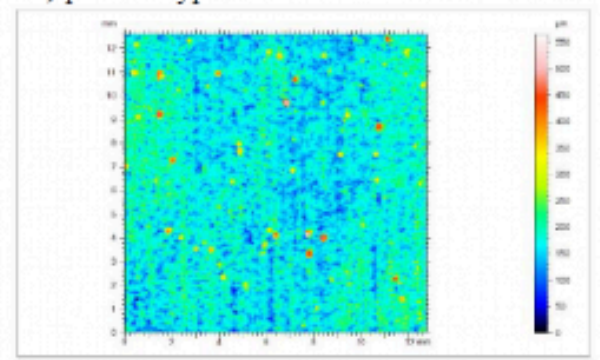

c2) powder type $3-\mathrm{S} 34$

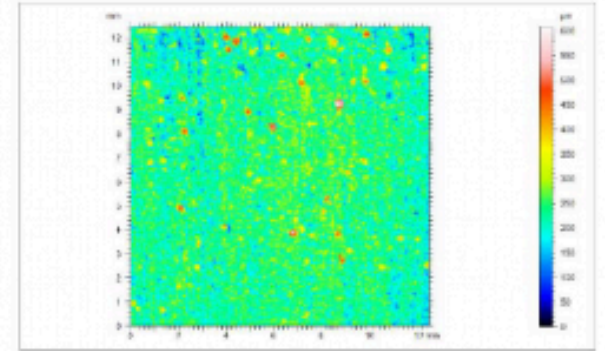

d2) powder type4 - H67

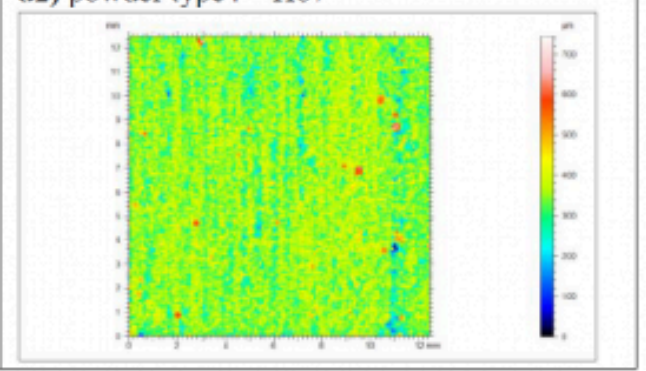

\begin{tabular}{c|c|c|c|c|c}
\hline Sample & & Type 1 - H6 & Type2 - H27 & Type3 -S34 & Type4-H67 \\
\hline \multirow{2}{*}{$\mathrm{S}_{2}(\mu \mathrm{m})$} & Top surface & 42.3 & $\mathbf{1 8 . 8}$ & $\mathbf{2 1 . 3}$ & 41.5 \\
\cline { 2 - 6 } & Side surface & 57.3 & $\mathbf{2 8 . 4}$ & $\mathbf{3 0}$ & 36.8 \\
\hline
\end{tabular}

Fig. 4 Color images of the top surface topography of parts produced by LPBF process $(\mathrm{P}=275 \mathrm{w}$ and $\mathrm{V}=700 \mathrm{~mm} / \mathrm{s})$ : (a1, a2) fine powder type1, (b1, b2) medium powder type2, (c1, c2) medium powder type3 and (d1, d2) coarse powder type 4 
Effect of powder size and processing parameters on surface, density and mechanical prop...

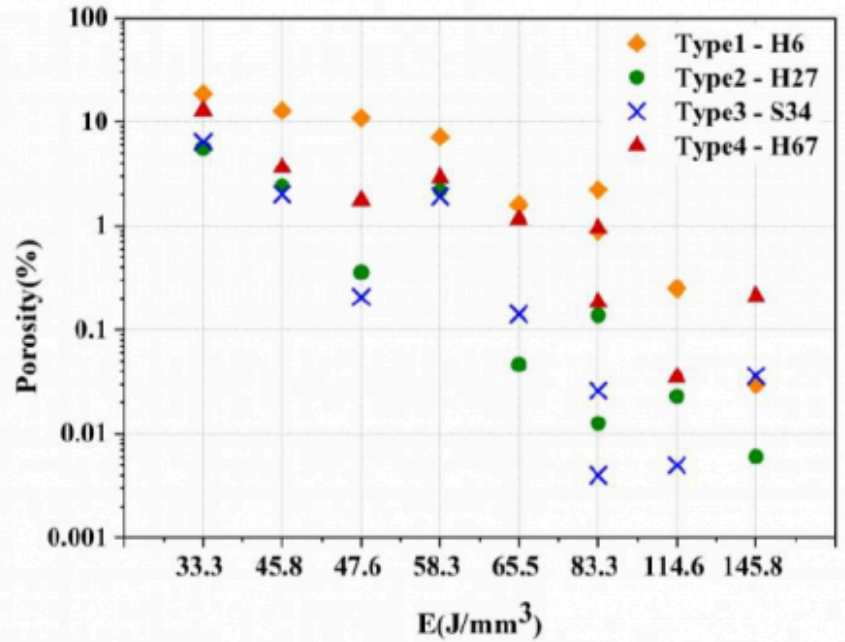

Fig. 5 Porosity as function of energy density for the four types of powder

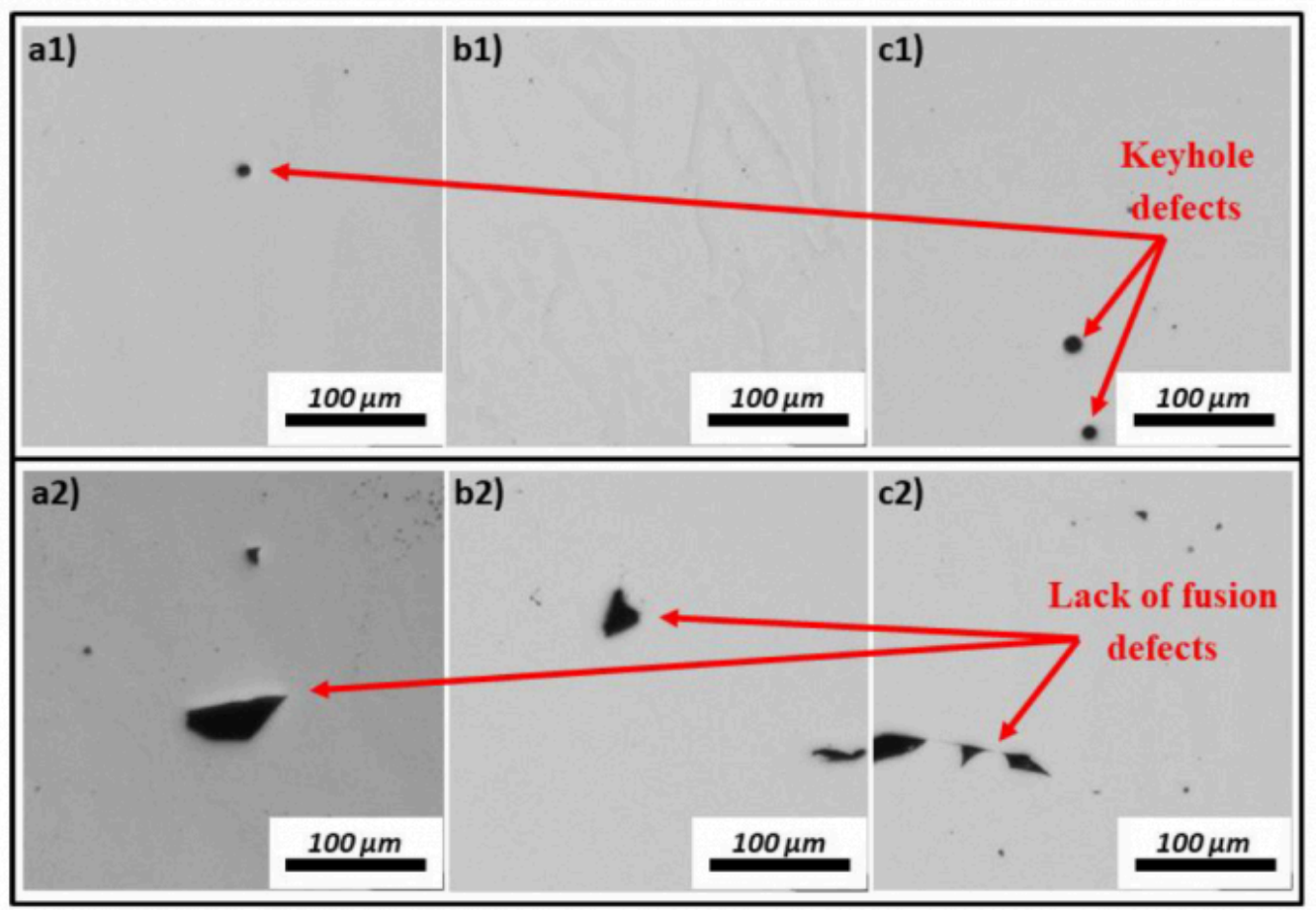

Fig. 6 Micrographs of non-etched cross-sections for samples produced with three powder types and different processing parameters: (a) Powder type2, (b) Powder type3 and (c) Powder type4. The laser power and scan speed are $350 \mathrm{~W}$ and $400 \mathrm{~mm} / \mathrm{s}$ for (1) and $275 \mathrm{~W}$ and $700 \mathrm{~mm} / \mathrm{s}$ for (2).

\subsection{Macro-hardness}

Macro-hardness results as function of the energy density are reported, in Fig. 7. The measured macro-hardness data 
showed good repeatability for dense samples with a maximum coefficient of variation of $4 \%$. It can be observed that regardless of the energy density, the SLM powder (type3) resulted in 30HV higher macro-hardness compared to Höganäs powders. This may be attributed to the differences in the chemical composition of powders (in particular for Si and Mn). The SLM powder type3 had higher Si and lower Mn percentages compared to the powders type2 and type3 from Höganäs. For all powder types, the macro-hardness followed an increasing trend when increasing the energy density and then stabilized for dense samples. The macro-hardness can be influenced by the porosities in the produced test parts. For low energy densities below $58.3 \mathrm{~J} / \mathrm{mm}^{3}$, the marco-hardness increased when increasing $\mathrm{E}$ which is due the decreasing of samples porosities. The interaction with sub-surface pores cannot be avoided within the indentation depth in samples with high porosities. Therefore, the macro-hardness and porosity are interconnected in the case low energy densities, as reported in [17].

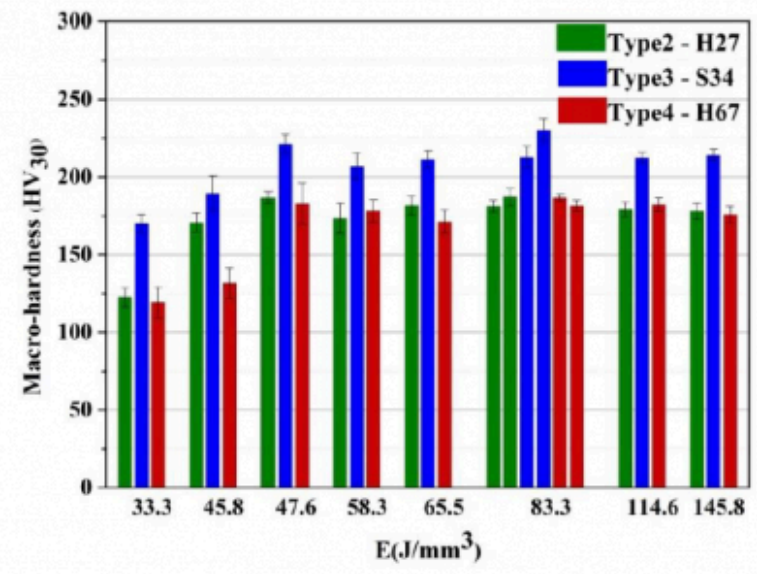

Fig. 7 Macro-hardness as function of energy density for medium and coarse powders

\section{Conclusion}

The aim of this study was to investigate the effect of powder particles size on the properties of SS 316L samples produced with LPBF process. Four different PSD were utilized to fabricate parts using various laser powers and scanning velocities. The samples, obtained with fine, medium and coarse powders, were compared based on the volumetric energy density parameter. The influence of powder properties and processing parameters on the porosity, top and side surface roughness and macro-hardness was studied. The main findings are summarized below:

1. Within the applied range of the volumetric energy density, the samples porosities decreased and then stabilized by increasing the energy density for all powder types. The samples fabricated with fine powder had higher porosities due to the powder poor flowability and its tendency to form agglomerates.

2. The minimum porosities were $0.03 \%, 0.005 \%$ and $0.035 \%$ for the fine, medium and coarse powder types, respectively. The lowest porosities were obtained for energy densities higher than $114 \mathrm{~J} / \mathrm{mm}^{3}$.

3. The fine powders had higher top and side surface roughness compared to medium and coarse powders. A difference of $5-70 \%$ between $S_{a}$ of fine powder parts and coarser counterparts were obtained. This is mainly due to the nonuniform spread powder layer. The lowest surface roughness is obtained using medium size powders ( $D_{50}=29 \mu \mathrm{m}$ and $\left.\mathrm{D}_{50}=41 \mu \mathrm{m}\right)$. No specific trend between surface roughness and energy densities was observed. 
Effect of powder size and processing parameters on surface, density and mechanical prop...

4. The macro-hardness increased when increasing the energy density up to $65.5 \mathrm{~J} / \mathrm{mm}^{3}$ which is inversely related to porosity. The type 3 powder had higher macro-hardness compared to type 2 and type4 powders. This might be attributed to the differences in powders chemical composition.

\section{Bibliography}

[1] T. DebRoy, H.L. Wei, J.S. Zuback, T. Mukherjee, J.W. Elmer, J.O. Milewski, A.M. Beese, A. Wilson-Heid, A. De, W. Zhang, Additive manufacturing of metallic components - Process, structure and properties, Prog. Mater. Sci. 92 (2018) 112224. https://doi.org/10.1016/j.pmatsci.2017.10.001.

[2] D. Bourell, J.P. Kruth, M. Leu, G. Levy, D. Rosen, A.M. Beese, A. Clare, Materials for additive manufacturing, CIRP Ann. - Manuf. Technol. 66 (2017) 659-681. https://doi.org/10.1016/j.cirp.2017.05.009.

[3] S.A.M. Tofail, E.P. Koumoulos, A. Bandyopadhyay, S. Bose, L. O’Donoghue, C. Charitidis, Additive manufacturing: scientific and technological challenges, market uptake and opportunities, Mater. Today. 21 (2018) 22-37. https://doi.org/10.1016/j.mattod.2017.07.001.

[4] A.B. Spierings, G. Levy, Comparison of density of stainless steel 316L parts produced with selective laser melting using different powder grades, n.d.

[5] A.B. Spierings, M. Voegtlin, T. Bauer, K. Wegener, Powder flowability characterisation methodology for powder-bedbased metal additive manufacturing, Prog. Addit. Manuf. (2016). https://doi.org/10.1007/s40964-015-0001-4.

[6] W.E. King, A.T. Anderson, R.M. Ferencz, N.E. Hodge, C. Kamath, S.A. Khairallah, A.M. Rubenchik, Laser powder-bed fusion additive anufacturing of metals; physics, computational, and materials challenges, in: Addit. Manuf. Handb. Prod. Dev. Def. Ind., CRC Press, 2017: pp. 461-506. https://doi.org/10.1201/9781315119106.

[7] F.-J. Gürtler, M. Karg, M. Dobler, S. Kohl, I. Tzivilsky, M. Schmidt, Influence of powder distribution on process stability in laser beam melting: Analysis of melt pool dynamics by numerical simulations, n.d.

[8] B. Liu, R. Wildman, C. Tuck, I. Ashcroft, R. Hague, INVESTIGATION THE EFFECT OF PARTICLE SIZE DISTRIBUTION ON PROCESSING PARAMETERS OPTIMISATION IN SELECTIVE LASER MELTING PROCESS, n.d.

[9] M.A. Balbaa, A. Ghasemi, E. Fereiduni, M.A. Elbestawi, S.D. Jadhav, J.P. Kruth, Role of powder particle size on laser powder bed fusion processability of AlSi10mg alloy, Addit. Manuf. (2020). https://doi.org/10.1016/ j.addma.2020.101630.

[10] L. Thijs, F. Verhaeghe, T. Craeghs, J.Van Humbeeck, J.P. Kruth, A study of the microstructural evolution during selective laser melting of Ti-6Al-4V, Acta Mater. 58 (2010) 3303-3312. https://doi.org/10.1016/j.actamat.2010.02.004.

[11] C. Meier, R. Weissbach, J. Weinberg, W.A. Wall, A.J. Hart, Critical influences of particle size and adhesion on the powder layer uniformity in metal additive manufacturing, J. Mater. Process. Technol. 266 (2019) 484-501. https://doi.org/10.1016/j.jmatprotec.2018.10.037.

[12] Y. He, A. Hassanpour, A.E. Bayly, Linking particle properties to layer characteristics: Discrete element modelling of cohesive fine powder spreading in additive manufacturing, Addit. Manuf. (2020) 101685. https://doi.org/10.1016/ j.addma.2020.101685.

[13] H. Chen, W. Yan, Spattering and denudation in laser powder bed fusion process: Multiphase flow modelling, Acta Mater. 196 (2020) 154-167. https://doi.org/10.1016/j.actamat.2020.06.033. 
[14] M. Yakout, M.A. Elbestawi, S.C. Veldhuis, Density and mechanical properties in selective laser melting of Invar 36 and stainless steel 316L, J. Mater. Process. Technol. 266 (2019) 397-420. https://doi.org/10.1016/ j.jmatprotec.2018.11.006.

[15] E. Liverani, S. Toschi, L. Ceschini, A. Fortunato, Effect of selective laser melting (SLM) process parameters on microstructure and mechanical properties of 316L austenitic stainless steel, J. Mater. Process. Technol. 249 (2017) 255-263. https://doi.org/10.1016/j.jmatprotec.2017.05.042.

[16] D. Sun, X. Li, W. Tan, A PARAMETRIC STUDY ON GRAIN STRUCTURE IN SELECTIVE LASER MELTING PROCESS FOR STAINLESS STEEL 316L, n.d.

[17] A.B. Spierings, N. Herres, G. Levy, Influence of the particle size distribution on surface quality and mechanical properties in additive manufactured stainless steel parts, in: 21st Annu. Int. Solid Free. Fabr. Symp. - An Addit. Manuf. Conf. SFF 2010, 2010: pp. 397-406.

PDF automatically generated on 2021-05-20 06:22:17

Article url: https://popups.uliege.be/esaform21/index.php?id=1563

published by ULiège Library in Open Access under the terms and conditions of the CC-BY License (https://creativecommons.org/licenses/by/4.0) 